\title{
Telemedicina Aplicada a la Valoración del Riesgo Cardiovascular: Experiencia en el Hospital María Angelines de Puerto Leguízamo, Putumayo
}

\section{Telemedicine Project Applied to the Assesment of Cardiovascular Risk Factors: Experience Gained in Maria Angelines Hospital, Puerto Leguizamo, Putumayo}

Recibido 15 Jun. 2013/Enviado para Modificación 18 Sep. 2013/Aceptado 20 Oct. 2013

\author{
Carlos Osorio López ${ }^{1}$ \\ Telemedicina de Colombia ITMS \\ Jeimy Cristancho Macías ${ }^{2}$ \\ Telemedicina de Colombia ITMS \\ Laura Pedreros Forero ${ }^{3}$ \\ Telemedicina de Colombia ITMS \\ Iván Martín Muñoz ${ }^{4}$ \\ Telemedicina de Colombia ITMS \\ Hernando Matiz Camacho ${ }^{5}$ \\ Telemedicina de Colombia ITMS \\ Rodolfo Vega Llamas ${ }^{6}$ \\ Telemedicina de Colombia ITMS
}

\section{RESUMEN}

Introducción: La telemedicina consiste en prestar servicios y soluciones médicas a distancia a una población determinada. Objetivo: Describir la valoración integral de los factores de riesgos cardiovasculares en el paciente con diagnóstico de Hipertensión Arterial (HTA) del proyecto de telemedicina. Materiales y métodos: Se realizó un estudio descriptivo en la población adulta que consulta al Hospital María Angelines del municipio de Puerto Leguízamo, departamento del Putumayo (Colombia); se tomó una muestra de 145 pacientes, que fueron diagnosticados por factores de riesgos asociados a la patología cardiovascular. Resultados: El género masculino promedió edades entre los 39 y 78 años; el género femenino se promedió entre los 40 y 80 años. La distribución por diagnósticos se dio de la siguiente manera: HTA más obesidad (70 casos), HTA más tabaquismo (68 casos), HTA más dislipidemia (80 casos), HTA más sedentarismo (110 casos), HTA más diabetes mellitus (50 casos). Del total de pacientes interconsultados (145), a 45 se les realizó monitoreo de presión ambulatoria, encontrando una distribución de diagnóstico y de clasificación de la enfermedad según los lineamientos y clasificación documentados por el VII JNC, así: estadio I, un total de 17 pacientes; estadio I-II, 18 pacientes; estadio II, 10 pacientes. Conclusión: La viabilidad de la valoración del riesgo cardiovascular en la modalidad de telemedicina contribuye al buen desarrollo de la práctica médica, y brinda la disponibilidad de una rehabilitación bajo parámetros específicos para cada paciente.

Palabras Clave: Telemedicina, hipertensión, enfermedades cardiovasculares (Fuente: DeCS).

\section{ABSTRACT}

Introduction: Telemedicine means to provide medical services and solutions to a specific population who live long distances from medical facilities. Purpose: To describe the integral assessment of cardiovascular risk factor in patient with a diagnosis of high blood pressure (HBP) of the Telemedicine Project. Materials and Methods: A descriptive study was focused on adult patient population that consult Maria Angelines Hospital from Puerto Leguizamo, Putumayo (Col). Results: Male gender average age between 39 and 78 years. Female gender average between 40 and 80 years. The distribution by diagnostic was as follows HTA more obesity (70 cases), HTA more smoking ( 68 cases), HTA more dyslipidemia ( 80 cases), HTA more sedentary life (110 cases), HTA more diabetes mellitus (50 cases). Of the total patients consulted to (145), to 45 monitoring ambulatory pressure, giving as a result a distribution of diagnosis and classification of the disease according to the classification and guidelines issued by the JNC VII, as well stage I a total of (17) patients Stage I-II, 18 patients. Conclusion: The assessment of viability of cardiovascular risk through telemedicine contributes to the good development of medical practice, and provides the availability of a rehabilitation based on specific parameters for each patient.

Keywords: telemedicine, hypertension, cardiovascular diseases (Source: MeSH, NLM).

\footnotetext{
${ }^{1}$ Médico. Coordinador científico Teleconsulta ITMS. e-mail: seoz8@ hotmail.com

${ }^{2}$ Médico. Director de Programa Riesgo Cardiovascular Hospital de Base

${ }^{3}$ Médico. Líder de entrenamiento nacional en uso de telesalud ITMS

${ }^{4}$ Enfermero. Coordinador operativo Teleconsulta ITMS

${ }^{5}$ Médico cardiólogo. Director científico ITMS

${ }^{6}$ Medico cardiólogo. Subdirector científico ITMS.
} 
Telemedicina Aplicada a la Valoración del Riesgo Cardiovascular:

Experiencia en el Hospital María Angelines de Puerto Leguízamo, Putumayo

\section{Introducción}

La telemedicina se define como la capacidad de brindar servicios médicos a distancia. Implica situaciones tan simples como solicitar vía telefónica una segunda opinión de un especialista sobre alguna patología concreta, o realizar un procedimiento quirúrgico mínimamente invasivo, cuyo operador resulta ser un robot sofisticado que se puede encontrar a kilómetros de distancia. La telemedicina es una rama de la medicina que va de la mano con las comunicaciones y con la telemática $(1,2)$, lo cual significa que es una de las herramientas tecnológicas más importantes para esta ciencia. El médico o el personal entrenado en los servicios de telemedicina tiene la oportunidad de realizar grandes contribuciones al desarrollo de la atención integral y con equidad de los pacientes, y así convertir la práctica médica en una labor con un alto índice de calidad, dadas las condiciones tecnológicas actuales (3).

Con esta herramienta, se ha logrado diseñar un modelo de atención en el que el médico general tiene la opción de interconsultar a todos sus pacientes mediante una plataforma científica, y de encontrar segundas opiniones en diagnósticos, tratamientos $\mathrm{y}$ rehabilitaciones. También permite conocer a los pacientes en su ambiente natural, y lograr valoraciones precisas de sus patologías. Con todo esto, se consigue, además, una relación más directa entre el médico general del nivel uno y dos, y las especialidades terapéuticas.

No obstante lo expresado hasta ahora y a pesar del gran avance de los últimos años en la informática y en las redes de interconexión entre ordenadores, la experiencia en la utilización de la telemedicina en la consulta médica es escasa. Para el caso que nos ocupa en particular, aunque existen algunos trabajos publicados en el campo cardiovascular $(4,5)$, en la actualidad, Telemedicina de Colombia (ITMS), como entidad prestadora de servicios médicos a distancia, es la única institución que realiza procedimientos especiales en las valoraciones integrales de los pacientes con hipertensión arterial.

Hacia Abril del año 2011, ITMS, en su ampliación de portafolio de servicios de su línea comercial y científica, incorporó a la plataforma de teleconsulta, la interconsulta por la especialidad de medicina interna, la cual contaba en sus inicios con un solo especialista en el área de la medicina del adulto, con un tiempo promedio de respuesta de 24 horas. Hoy en día, gracias a la acogida y demanda de teleconsultas en esta área por las instituciones prestadoras de servicios de salud, el departamento cuenta con dos profesionales de amplia experiencia en el área cuyas respuestas no sobrepasan las 12 horas. Justamente, el objetivo del presente trabajo consiste en evaluar la utilidad y viabilidad de la telemedicina en el seguimiento de los pacientes con enfermedad crónica hipertensiva $\mathrm{y}$ factores de riesgo asociados, desde la atención primaria, teniendo en cuenta la posición geográfica del municipio de Puerto Leguízamo (Putumayo) y todo lo que significa para un paciente su traslado hasta el sitio que le designe su aseguradora para la consulta médica con el especialista, lo que en sí conlleva elevados gastos y recursos de personal.

En el siguiente artículo, se expondrá en concreto la experiencia que constituyó la valoración de los factores de riesgos asociados a la patología hipertensiva de la población de Puerto Leguizamo (Putumayo), bajo la modalidad de teleconsulta asincrónica, con el departamento de medicina interna del centro de referencia en telemedicina, es decir, con Telemedicina de Colombia (ITMS).

\section{Materiales y Métodos}

La investigación se realizó bajo la forma de un estudio transversal, en el cual se realizó seguimiento a los pacientes con diagnóstico de hipertensión arterial asociados a factores de riesgo cardiovascular del programa de Atención Integral al Paciente con Patología Crónica no Infecciosa del Hospital María Angelines, del municipio de Puerto Leguízamo, departamento del Putumayo. Dicho programa cuenta con vigencia de 1 año desde su instauración, y en la actualidad tiene un registro de 200 pacientes en el club de hipertensos, el cual fue el criterio de inclusión primordial para determinar la población en este estudio. La cobertura de los derechos en atención en salud de estos adultos está dada por diferentes aseguradoras, tanto del régimen contributivo como del régimen subsidiado, pero también algunos aparecen vinculados a la secretaria de salud del departamento. Durante el análisis, se encontraron actividades de promoción y de prevención, referidas a la modificación del estilo de vida. Con ello se busca la reducción del riesgo en los pacientes con clasificación de riesgo cardiovascular bajo y las charlas de

Cienc. innov. salud. Diciembre 2013; 1 (2): 95 - 100. Universidad Simón Bolívar (Col). ISSN: 2344-8636 
capacitación al paciente con riesgo cardiovascular alto en el buen uso de la medicación, la importancia de los controles y las reducciones de factores de riesgos contribuyentes como el stress y el sedentarismo, que potencialmente alteran el estado de salud desde el punto de vista cardiovascular (6).

En el flujo de actividades técnico-operativas y técnico-científicas, se realizó una valoración previa por médico general, mediante la que se revisó cada caso de manera puntual. Fue así como se actualizó la historia clínica, y se enfocó la consulta en encontrar la asociación patológica de base con factores de riesgos cardiovasculares asociados, según el modelo de tamizaje del estudio Framinghan, el cual determina el riesgo de enfermedad cardiovascular a 10 años. En este punto, es de vital importancia aclarar que los factores de riesgo se dividen en dos categorías: principales y contribuyentes. Se designan como factores de riesgo principales a aquellos sobre los cuales se ha comprobado que aumentan el riesgo cardiovascular. En cambio, los factores contribuyentes son aquellos que pueden aumentar el riesgo cardiovascular, pero cuyo papel exacto no se ha definido aún.

Cuantos más factores de riesgo tenga una persona, mayores serán sus probabilidades de padecer una enfermedad del corazón. Algunos factores de riesgo pueden cambiarse, tratarse o modificarse, pero otros, no. Sin embargo, es claro que el control sobre el mayor número posible de factores de riesgo, mediante cambios en el estilo de vida y/o medicamentos, puede reducir el riesgo cardiovascular. Los más comunes son la edad, los hábitos saludables de vida; la actividad física, el tabaquismo, el alcoholismo, dieta rica en sal, factores hereditarios, dislipidemia asociada, síntomas relacionados con estado menopáusico, estrés y diabetes mellitus. En prevención primaria, el objetivo básico es conseguir que el sujeto elimine o mantenga control sobre estos factores asociados y alcance y conserve valores de presión arterial inferiores a $140 / 90$, un colesterol total inferior a $200 \mathrm{mg} / \mathrm{dL}(190$ $\mathrm{mg} / \mathrm{dl}$ en guías europeas) y un LDLc inferior a 130 $\mathrm{mg} / \mathrm{dL}(115 \mathrm{mg} / \mathrm{dl}$ en las guías europeas). También se busca que el paciente tenga un peso adecuado, practique actividad física y limite el consumo de grasas saturadas y de colesterol en la dieta.

En específico, una dieta adecuada, la eliminación del tabaco y la realización de una actividad física, acorde con la edad y la condición física de la persona, pueden reducir el riesgo de enfermedad isquémica coronaria. De ahí la necesidad de estructurar programas tendientes a que la población general adopte dichos hábitos, lo cual se convierte en un aspecto fundamental para las personas quienes que no han desarrollado una prevención primaria (ECV), pero fuman, tienen dislipemia, diabetes o hipertensión, al igual que quienes son obesos, sedentarios y cuyos familiares, en primer grado, hayan presentado una enfermedad coronaria precoz.

La plataforma utilizada para la realización de la teleconsulta en el Hospital Maria Angelines es la red de Internet con enlace satelital. Para la generación de las teleconsultas, el médico en terreno cuenta con una estación de telemedicina, la cual tiene todos los dispositivos para la creación de teleconsultas en línea: contiene un ordenador portátil de última generación, impresora, dermatoscopio digital, cámaras digitales para videoconferencias y dispositivos para la toma de signos vitales. La consulta es llevada a cabo desde esta estación en un formato de historia clínica digital, que cuenta con la posibilidad de anexar estudios paraclínicos y fotos de cada uno de los pacientes. Esta historia clínica es enviada hasta el centro de referencia en Bogotá, donde es valorada por el médico general para la aprobación o devolución en caso de no contar con las necesidades tecnológicas o científicas para resolver el caso. Si cumple, en cambio, con todos los requisitos, se envía al especialista. Es de mucha importancia resaltar que todo este flujo de información se mantiene bajo protección, por claves de acceso a la plataforma que solo conocen las personas que cuentan con un vínculo científico en la institución.

En forma previa a la puesta en marcha del proyecto, los médicos en terreno reciben capacitación por parte de coordinadores científicos, y realizan operativos a cargo del área de teleconsulta del ITMS. Este hecho garantiza el soporte y el buen uso de la plataforma desde todo punto de vista, y asegura la valoración de los pacientes de manera integral, el cual es el objetivo fundamental de esta.

\section{Resultados}

El Hospital María Angelines maneja una población de 200 pacientes con diagnostico establecido de hipertensión arterial, los cuales fueron valorados por médico general. De estos, se interconsultaron en plataforma de telemedicina de ITMS un total de 145

Cienc. innov. salud. Diciembre 2013; 1 (2): 95 - 100. Universidad Simón Bolívar (Col). ISSN: 2344-8636 
Telemedicina Aplicada a la Valoración del Riesgo Cardiovascular:

pacientes, sobre quienes el médico generalista de eventos telemédicos planteó la necesidad de consultar una segunda opinión por parte de medicina especializada, dada la complejidad de algunos casos y, en otros, por cumplir la normatividad vigente de salud pública en lo referente al sistema integral de promoción y prevención de las enfermedades crónicas no infecciosas.

La herramienta que se empleó para la predicción de eventos cardiovasculares fue la calculadora de Framingham (6), con su actualización versión 2008. A partir de ella, se logró encontrar en toda la población objeto de estudio un factor primario no modificable de riesgo cardiovascular, que es el de hipertensión arterial con una cifra de 145 pacientes, e hipertensión más diabetes mellitus en un total de 80 pacientes, los cuales fueron prioridad dada su estándar de clasificación que se traduce en un valor de riesgo cardiovascular alto cuando se asocia a la edad y los factores de comorbilidad. Este fue entonces el grupo que se priorizó al momento de las teleconsultas.

La distribución de los factores de riesgo asociados a la patología cardiovascular medidos en la población tiene como variables de inclusión a los pacientes que se encontraban en un rango de edad entre los 20 y 85 años; dando como resultado una variable descriptiva de la siguiente forma: género masculino, con un promedio de edades entre los 39 y 78 años, hipertensión estadio I-II; y en el género femenino, con promedió entre los 40 y 80 años, hipertensión estadio I-II-II. Esto convierte a las mujeres de la población en candidatas potenciales a padecer compromisos vasculares más frecuentes en comparación con los hombres, según la clasificación del séptimo comité. La distribución por diagnósticos, ajustando factores de riesgo modificables y no modificables, se dio de la siguiente manera: hipertensión arterial más obesidad, 70 casos; hipertensión arterial más tabaquismo, 68 casos; hipertensión arterial más dislipidemia, 80 casos; hipertensión más sedentarismo, 110 casos; hipertensión arterial más diabetes mellitus, 80 casos. En la tabla 2, se muestra una descripción detallada de los resultados de los 145 pacientes interconsultados.

Del total de pacientes interconsultados (145), a 45 se les realizó monitoreo de presión ambulatoria, encontrando una distribución de diagnóstico $\mathrm{y}$ de clasificación de la enfermedad según los lineamientos y clasificación documentados por el VII Joint National Committe on Prevention (7), así: estadio I, un total de
17 pacientes; estadio I-II, 18 pacientes; estadio II, 10 pacientes; también se hicieron exámenes que registraron normalidad en 9 pacientes de la muestra. El resto de la clasificación se realizó a través del método tradicional por parte del médico cardiólogo de la institución, antes que se acudiera al monitoreo de presión con lectura; sin embargo, estos hallazgos brindaron la disponibilidad de una valoración integral del estado y del riesgo cardiovascular de los pacientes, porque gracias al MAPA (Monitoreo Ambulatorio de Presión Arterial), se logró un ajuste de parte del médico especialista y del médico general en la medicación del paciente.

El tiempo de recolección de información abarcó el periodo comprendido entre los meses de febrero a junio del año 2013, en los cuales se estableció la valoración del riesgo cardiovascular bajo la modalidad de telemedicina, contribuyendo al buen desarrollo de la práctica médica, y presentándose la disponibilidad de una rehabilitación en parámetros específicos para cada paciente. También se entregó una valoración personalizada, a partir de los datos antropométricos y de factores de riesgos asociados a otro tipo de morbilidad. De esta forma, se logró contribuir al buen desarrollo de las metas de los planes de salud pública, que en la actualidad se exige a los hospitales en lo que concierne a las estrategias de atención a la población crónica con diagnóstico de hipertensión.

Tabla 1. Diagnostico realizados

\begin{tabular}{lc}
\hline \multicolumn{1}{c}{ DIAGNÓSTICOS } & TOTAL \\
\hline Hipertensión Arterial & 145 \\
Hipertensión Arterial más Obesidad & 80 \\
Hipertensión Arterial más Tabaquismo & 68 \\
Hipertensión Arterial más Dislipidemia & 80 \\
Hipertensión Arterial más Sedentarismo & 110 \\
Hipertensión arterial más diabetes mellitus & 80 \\
\hline
\end{tabular}

Teniendo como base la clasificación del riesgo cardiovascular según la escala de Framinghan, la patología de base más frecuentemente registrada es el estadio hipertensivo, que, asociado al resto de factores, nos entrega una clasificación de la población de estudio en tasas representativas con 30 casos de riesgo cardiovascular (RC) muy alto, 50 casos de RC alto, 40 casos de RC medio o moderado, y 15 casos de RC bajo. Así fue como se estableció una base de datos actualizada sobre los pacientes a los cuales se le debe instaurar un seguimiento más controlado y ajustar la

Cienc. innov. salud. Diciembre 2013; 1 (2): 95 - 100. Universidad Simón Bolívar (Col). ISSN: 2344-8636 
disponibilidad a la reducción de mortalidad cardiovascular, teniendo en cuenta que los pacientes del grupo poblacional estudiado tienen índices de morbilidad elevados.

En el análisis de los resultados, se encontró que la población adulta que presenta patología crónica con factores de riesgo cardiovascular es significativa si se toman como base los datos con los que contaba inicialmente el Hospital María Angelines (8) y no el total registrado por el DANE (Departamento Administrativo Nacional de Estadísticas) que, para este caso, representó un $72,5 \%$ teniendo en cuenta el conjunto de variables asociadas. Por este motivo, para los planes de intervención de este fenómeno en salud pública, se hace prioritaria la mejora a nivel nacional con programas de atención que identifiquen, clasifiquen e intervengan a los usuarios de forma oportuna, para dar cumplimiento de esta forma a los requerimientos que en la actualidad se adelantan por parte del Ministerio de Salud y Protección Social. Cabe resaltar la actitud que se tiene por parte del personal asistencial de la institución en la mejora de la atención y la puesta en marcha de un protocolo de atención integral a esta población. El impacto en la gestión y la evaluación de la misma cuenta con una vigencia de 1 año, tiempo en que se han de analizar las pirámides de morbimortalidad, considerando que lo que se espera es la reducción de estos índices en la comunidad hipertensa de esta población.

Es importante resaltar que la acción terapéutica en esta población está ligada al componente de reglamentación vigente, según el Plan Obligatorio de Salud. Se ha encontrado variedad en la entrega de medicamentos, debido a los protocolos establecidos por las entidades aseguradoras de las cuales depende dicho suministro. Lo anterior significa que, a nivel del impacto poblacional, se encuentra diversidad en los resultados referidos al componente científico, al no existir un ajuste estandarizado en la medicación, según los reportes actuales de medicina basada en la evidencia, y las guías de manejo recomendadas para este tipo de patologías.

\section{Discusión}

La atención del paciente hipertenso con factores de riesgo asociados a cargo de una plataforma de teleconsulta por telemedicina ofrece la posibilidad de realizar una valoración interdisciplinar en un tiempo oportuno de respuestas, durante el cual se logran tomar acciones tendientes a corregir el estado patológico (9). La integración y el nivel de resolución demuestran que la atención inicial puede ser guiada y elaborada por un profesional de la salud con capacidad de diagnóstico, en este caso un médico general, el cual tiene todas las facultades para establecer un protocolo de rehabilitación cardiovascular utilizando la herramienta de teleconsulta por medicina interna, cardiología, endocrinología, nutrición y dietética.

Cabe anotar que una parte fundamental del manejo de este tipo de patología va de la mano del entrenamiento y la incorporación de los profesionales que se encuentren al cuidado y control del paciente. Ello constituye una estrategia válida que genera un control de la enfermedad crónica no trasmisible del paciente, reduciendo el ingreso a los servicios de urgencias por alteración aguda cardiovascular $\mathrm{y}$, por otra parte, contribuye sustancialmente al bienestar del adulto, mejorando su calidad de vida y brindándole más tiempo de servicio útil a la sociedad (10).

En la evaluación del proyecto de telemedicina, se han identificado una serie de beneficios que muestran un impacto sanitario positivo en la población de su área de influencia, pero algunos beneficios no han sido valorados hasta el momento, tales como el ahorro en costos por los servicios que se les brinda a estos pacientes y los traslados de control por medicina especializada (11).

La reducción de las patologías del adulto apunta a mejorar los objetivos de reducción de la mortalidad de salud pública trazados para el milenio, a nivel de la Organización Panamericana de la Salud y el Ministerio de Salud y Protección Social de Colombia, mediante una estrategia de impacto y beneficio hacia este tipo de población, que a la vez resulta resolutiva y de bajo costo para los hospitales (12). Por otro lado, el proyecto de telemedicina también repercute positivamente en el Plan Fronteras para la Prosperidad que adelanta el Ministerio de Relaciones Exteriores (13) y que busca llevar recursos tecnológicos en pro de la valoración del paciente de la zona limítrofe del país, entregando herramientas para el desarrollo de actividades médicas que contribuyan al control y al buen funcionamiento de los programas de prevención y promoción de las instituciones hospitalarias de las zonas de frontera. 
Telemedicina Aplicada a la Valoración del Riesgo Cardiovascular:

En la actualidad, las políticas de salud pública para la prevención de la enfermedad cardiovascular y el abordaje desde la implementación de sistemas integrados en telemedicina constituyen, entonces, una oportunidad de generar un manejo integral $y$ multidisciplinar del paciente en este tipo de patologías crónicas, contribuyendo a una atención medica con equidad, lo cual es uno de los pilares fundamentales de los sistema de telemedicina.

\section{Referencias}

1. García BM. El valor educativo de la telemedicina. Educ. méd. 2006; 9 (Suppl 2): 38-43.

2. Monteagudo JL, Serrano L, Hernández SC. La telemedicina: ¿ciencia o ficción? Anales Sis San Navarra. 2005; 28(3): 309-323.

3. Matiz $\mathrm{CH}$. El futuro de la medicina con equidad en Colombia: La Telemedicina. Rev. Col. Cardiol. 2007; 14 (1): 1-8.

4. Giuliano I, Barcellos C, Von Wangenheim A, Coutinho $\mathrm{M}$. Issuing electrocardiographic reports remotely: experience of the telemedicine network of Santa Catarina. Arq. Bras. Cardiol. 2012; 99 (5): 1023-1030.

5. Costa AM, Carvalho M, Calviño J, Sousa M, Sousa G, Gaspar E et al. Ecocardiografia por telemedicina em recém-nascidos num hospital de nível II: casuística de quatro anos. Nascer e Crescer. 2011; 20(3): 137-140.

6. Dawber TR, Gilcin FM, Moore F. National Heart Institute, National Institutes of Health, Public Health Service, Federal Security Agency, Washington, D. C., Epidemiological approaches to heart disease: The Framingham study presented at a joint session of the epidemiology, health officers, medical care, and statistics sections of the American Public Health Association, at the Seventy-eighth Annual Meeting in St. Louis, Mo., 1950.

7. U.S. Department of health and human services. National Institutes of Health. National Heart, Lung, and Blood Institute. The seventh report of the joint national committee on prevention, detection, evaluation, and treatment of high blood pressure. 2004.

8. Hospital María Angelines. Departamento de estadística de Puerto Leguízamo, Putumayo, datos del año 2011-2012-2013.
9. Prescher S, Deckwart O, Winkler S, Koehler K, Honold M, Koehler F. Telemedical care: feasibility and perception of the patients and physicians: a survey-based acceptance analysis of the Telemedical Interventional Monitoring in Heart Failure (TIM-HF) trial. Eur J Prev Cardiol. 2013; 20 (2): 18-24.

10. Munro J, Angus N, Leslie SJ. Patient focused Internet-based approaches to cardiovascular rehabilitation - a systematic review. J Telemed Telecare. 2013; 19(6):347-353.

11. Horwood T, Aylott M, Loyola M, Henderson J, Frank G, Fyfe ML. Telecardiology on Vancouver Island: imagination to implementation. Stud Health Technol Inform. 2013; 183: 63-67.

12. Sociedad Colombiana de Cardiología, Guías clínicas de hipertensión arterial. Bogotá, 2007.

13. Ministerio de Relaciones Exteriores. Plan Fronteras para la Prosperidad. Disponible en: http://www.cancilleria.gov.co/prosperity/presentat ion. 